\title{
Motion in rotating Earth: exact solutions and second-order effects of noninertial forces
}

\author{
Eduardo Moraes Diniz ${ }^{*}$ \\ ${ }^{1}$ Universidade Federal do Maranhão, Departamento de Física, São Luís, MA, Brasil.
}

\begin{abstract}
Received on October 15, 2021. Revised on January 12, 2022. Accepted on January 18, 2022.
The motion of a particle evaluated from the noninertial frame of the rotating Earth with constant angular velocity $\omega$ is solved analytically without carrying out a priori approximations, that is, considering both the Coriolis and the centrifugal terms. Position vector solutions written in terms of generic initial conditions were obtained from a decoupled sixth-order equation of motion, obtained using a fully vectorial approach, without specifically resorting to coordinates. By comparing the exact solutions for the complete and approximate (ignoring the centrifugal term from the beginning) problems, great differences can be seen in the order of the decoupled differential equation as well as in the temporal dependencies of the noninertial terms of the solutions. Noninertial effects for the solution were classified according to the order of magnitude in $\omega$, where first-order effects are oriented east-west and the second-order ones are oriented north-south.

Keywords: Noninertial frames, Noninertial effects, Second-order effects, Motion in rotating Earth.
\end{abstract}

\section{Introduction}

The motion of particles in noninertial frames is often studied in undergraduate Classical Mechanics courses. A special part of this topic deals with motion evaluated from a fixed frame on Earth, whose equation of motion is given by 1

$$
\ddot{\boldsymbol{r}}=\boldsymbol{g}_{0}+\boldsymbol{\omega} \times(\boldsymbol{R} \times \boldsymbol{\omega})+2 \dot{\boldsymbol{r}} \times \boldsymbol{\omega}+\boldsymbol{\omega} \times(\boldsymbol{r} \times \boldsymbol{\omega})+\boldsymbol{r} \times \dot{\boldsymbol{\omega}}
$$

where $\boldsymbol{r}$ is the position vector, $\boldsymbol{g}_{0}$ is the Earth's constant gravitational field, $\boldsymbol{\omega}$ is the vector that gives the orientation of the Earth's rotation, and $\boldsymbol{R}$ is the vector radius that connects the planet's center (origin of the inertial frame) and the noninertial frame located on its surface. In the equation of motion (1), the noninertial terms couple the Cartesian coordinates and come in the form of vector products being a correction to the gravitational field due to the centrifugal effect, Coriolis, centrifugal and azimuthal or Euler accelerations [2]. A common consideration is to neglect the variation of the Earth's rotation, $\dot{\omega}=\mathbf{0}$, and then define the field $\boldsymbol{g}=\boldsymbol{g}_{0}+\boldsymbol{\omega} \times(\boldsymbol{R} \times \boldsymbol{\omega})$ as a constant gravity field [1, 3]. The equation of motion (1) is then

$$
\ddot{\boldsymbol{r}}=\boldsymbol{g}+2 \dot{\boldsymbol{r}} \times \boldsymbol{\omega}+\boldsymbol{\omega} \times(\boldsymbol{r} \times \boldsymbol{\omega})
$$

Among the noninertial forces, the Coriolis force is certainly the most investigated because of its effects such

\footnotetext{
* Correspondence email address: eduardo.diniz@ufma.br
}

as the deviation to the right (left) of objects moving in the Northern (Southern) Hemisphere. Coriolis force also contributes to the counterclockwise cyclonal movement of air masses in the Northern Hemisphere and clockwise in the Southern Hemisphere among others [1 3 .

The justification for the emphasis given to the Coriolis force is that it is proportional to $\omega$, or firstorder in $\omega$, which is symbolically represented by $\mathcal{O}(\omega)$, being more relevant than the centrifugal term, which is $\mathcal{O}\left(\omega^{2}\right)$, because of the slow terrestrial rotation of $\omega=$ $\frac{2 \pi}{24}\left(\frac{366.5}{365.5}\right) \mathrm{rad} / \mathrm{h} \approx 7.3 \cdot 10^{-5} \mathrm{rad} / \mathrm{s}$, where the term in parentheses is a correction factor evaluated as the ratio between the number of sidereal days and the number of solar days so that $\omega$ is determined from the fixed stars 3. However, when the approximation of small $\omega$ is applied directly to the equation of motion (2), despite being correct in determining the noninertial effects, it leads to equations of motion for the phenomenon that are completely different from those that are found by considering all the terms of equation (2), as I will show later. Furthermore, the neglecting of $\mathcal{O}\left(\omega^{2}\right)$ terms directly from the equation of motion (2) prevents the correct analysis of effects that depend on this order of magnitude. Thus, even if one recognizes the relevant role, albeit a supporting one, of the $\mathcal{O}\left(\omega^{2}\right)$ terms, their consequences are generally treated in a qualitative or semi-quantitative way 2 .

In the next section vector solutions of equation (2) are obtained with and without the centrifugal term. The third section is devoted to show the second-order effects obtained from the complete problem. 


\section{Decoupling and Solution of Equations of Motion}

\subsection{Coriolis and centrifugal}

Equation 2 is coupled in their Cartesian coordinates, once $\ddot{x}$ depends on $y$ and $z$ as well as their time derivatives due to the vector products originated from non-inertial terms. The same happens for $\ddot{y}$ and $\ddot{z}$. To decouple the equation of motion (2) I will develop a fully vector approach. To do this, two expressions will be useful:

$$
\left\{\begin{aligned}
& \ddot{\boldsymbol{r}} \times \boldsymbol{\omega}=\boldsymbol{g} \times \boldsymbol{\omega}-2 \boldsymbol{\omega} \times(\dot{\boldsymbol{r}} \times \boldsymbol{\omega})+\omega^{2} \boldsymbol{r} \times \boldsymbol{\omega} \\
& \boldsymbol{\omega} \times(\ddot{\boldsymbol{r}} \times \boldsymbol{\omega})= \boldsymbol{\omega} \times(\boldsymbol{g} \times \boldsymbol{\omega})+2 \omega^{2} \dot{\boldsymbol{r}} \times \boldsymbol{\omega} \\
&+\omega^{2} \boldsymbol{\omega} \times(\boldsymbol{r} \times \boldsymbol{\omega})
\end{aligned}\right.
$$

both obtained from equation (2) and calculated using the vector identity $\boldsymbol{a} \times(\boldsymbol{b} \times \boldsymbol{c})=(\boldsymbol{a} \cdot \boldsymbol{c}) \boldsymbol{b}-(\boldsymbol{a} \cdot \boldsymbol{b}) \boldsymbol{c}$ when convenient. Successively differentiating equation (2) with respect to time, one obtains expressions from $\dddot{\boldsymbol{r}}$ to $\boldsymbol{r}^{(6)}$, as written in the system of equations (4)

$$
\left\{\begin{array}{l}
\dddot{\boldsymbol{r}}=2 \boldsymbol{g} \times \boldsymbol{\omega}-3 \boldsymbol{\omega} \times(\dot{\boldsymbol{r}} \times \boldsymbol{\omega})+2 \omega^{2} \boldsymbol{r} \times \boldsymbol{\omega} \\
\boldsymbol{r}^{(4)}=-3 \boldsymbol{\omega} \times(\boldsymbol{g} \times \boldsymbol{\omega})-4 \omega^{2} \dot{\boldsymbol{r}} \times \boldsymbol{\omega}-3 \omega^{2} \boldsymbol{\omega} \times(\boldsymbol{r} \times \boldsymbol{\omega}) \\
\boldsymbol{r}^{(5)}=-4 \omega^{2} \boldsymbol{g} \times \boldsymbol{\omega}+5 \omega^{2} \boldsymbol{\omega} \times(\dot{\boldsymbol{r}} \times \boldsymbol{\omega})-4 \omega^{4} \boldsymbol{r} \times \boldsymbol{\omega} \\
\boldsymbol{r}^{(6)}=5 \omega^{2} \boldsymbol{\omega} \times(\boldsymbol{g} \times \boldsymbol{\omega})+6 \omega^{4} \dot{\boldsymbol{r}} \times \boldsymbol{\omega}+5 \omega^{4} \boldsymbol{\omega} \times(\boldsymbol{r} \times \boldsymbol{\omega})
\end{array}\right.
$$

where for each calculated derivative I used the expressions for the vector products $\ddot{\boldsymbol{r}} \times \boldsymbol{\omega}$ and $\boldsymbol{\omega} \times(\ddot{\boldsymbol{r}} \times \boldsymbol{\omega})$ written in (3) in order to make the right side of the system of equations (4) dependent on vector products of $\boldsymbol{r}$ and $\dot{\boldsymbol{r}}$ with $\boldsymbol{\omega}$. This is interesting for expressing the general solution in terms of initial conditions.

The last equation of (4) is ready to be decoupled. To do this, the products $\dot{\boldsymbol{r}} \times \boldsymbol{\omega}$ and $\boldsymbol{\omega} \times(\boldsymbol{r} \times \boldsymbol{\omega})$ must be written in terms of the derivatives $\ddot{\boldsymbol{r}}$ and $\boldsymbol{r}^{(4)}$ using the equation of motion (2) and the second equation of the system (4), respectively. By substituting such expressions in the equation for $\boldsymbol{r}^{(6)}$ in the system (4), it results in a decoupled sixth-order differential equation, i.e. a differential equation that does not exhibit any vector product of $\boldsymbol{r}$ or $\dot{\boldsymbol{r}}$ :

$$
\boldsymbol{r}^{(6)}+2 \omega^{2} \boldsymbol{r}^{(4)}+\omega^{4} \ddot{\boldsymbol{r}}=\omega^{4} \boldsymbol{g}-\omega^{2} \boldsymbol{\omega} \times(\boldsymbol{g} \times \boldsymbol{\omega})
$$

where its general solution can be obtained from standard methods for solving ordinary differential equations: it is a sum of a complementary general solution of the homogeneous form of (5), obtained from the roots of its characteristic polynomial, and a particular solution of the inhomogeneous problem. Therefore, the general solution of (5) can be written as

$$
\begin{aligned}
\boldsymbol{r}(t)= & \boldsymbol{c}_{0}+\boldsymbol{c}_{1} t+\frac{1}{2} \boldsymbol{g} t^{2}-\frac{\boldsymbol{\omega} \times(\boldsymbol{g} \times \boldsymbol{\omega}) t^{2}}{2 \omega^{2}} \\
& +\left(\boldsymbol{c}_{2}+\boldsymbol{c}_{3} t\right) \cos (\omega t)+\left(\boldsymbol{c}_{4}+\boldsymbol{c}_{5} t\right) \sin (\omega t)
\end{aligned}
$$

where $\boldsymbol{c}_{j}$ are arbitrary vector constants. To find the general solution in terms of generic initial conditions $\boldsymbol{r}(0)=\boldsymbol{r}_{0}$ and $\dot{\boldsymbol{r}}(0)=\boldsymbol{v}_{0}$ it is necessary to use the coupled equation of motion (2) in addition to the first three equations of system (4). By doing so, one finds a set of six vector equations from $\boldsymbol{r}(0)$ to $\boldsymbol{r}^{(5)}(0)$ that can be solved exactly by producing the following expressions for the $\boldsymbol{c}_{j}$ :

$$
\left\{\begin{array}{l}
\boldsymbol{c}_{0}=\boldsymbol{r}_{0}-\frac{\boldsymbol{\omega} \times\left(\boldsymbol{r}_{0} \times \boldsymbol{\omega}\right)}{\omega^{2}}-\frac{\boldsymbol{\omega} \times(\boldsymbol{g} \times \boldsymbol{\omega})}{\omega^{4}} \\
\boldsymbol{c}_{1}=\boldsymbol{v}_{0}-\frac{\boldsymbol{\omega} \times\left(\boldsymbol{v}_{0} \times \boldsymbol{\omega}\right)}{\omega^{2}} \\
\boldsymbol{c}_{2}=\frac{\boldsymbol{\omega} \times\left(\boldsymbol{r}_{0} \times \boldsymbol{\omega}\right)}{\omega^{2}}+\frac{\boldsymbol{\omega} \times(\boldsymbol{g} \times \boldsymbol{\omega})}{\omega^{4}} \\
\boldsymbol{c}_{3}=-\boldsymbol{r}_{0} \times \boldsymbol{\omega}-\frac{\boldsymbol{g} \times \boldsymbol{\omega}}{\omega^{2}}+\frac{\boldsymbol{\omega} \times\left(\boldsymbol{v}_{0} \times \boldsymbol{\omega}\right)}{\omega^{2}} \\
\boldsymbol{c}_{4}=\frac{\boldsymbol{r}_{0} \times \boldsymbol{\omega}}{\omega}+\frac{\boldsymbol{g} \times \boldsymbol{\omega}}{\omega^{3}} \\
\boldsymbol{c}_{5}=\frac{\boldsymbol{\omega} \times\left(\boldsymbol{r}_{0} \times \boldsymbol{\omega}\right)}{\omega}+\frac{\boldsymbol{v}_{0} \times \boldsymbol{\omega}}{\omega}+\frac{\boldsymbol{\omega} \times(\boldsymbol{g} \times \boldsymbol{\omega})}{\omega^{3}}
\end{array}\right.
$$

that when replaced in the general solution (6) result in

$$
\begin{aligned}
\boldsymbol{r}(t)= & \boldsymbol{r}_{0}+\boldsymbol{v}_{0} t+\frac{1}{2} \boldsymbol{g} t^{2} \\
& +\frac{\sin (\omega t)-\omega t \cos (\omega t)}{\omega} \boldsymbol{r}_{0} \times \boldsymbol{\omega} \\
& +\frac{t \sin (\omega t)}{\omega} \boldsymbol{v}_{0} \times \boldsymbol{\omega} \\
& +\frac{\sin (\omega t)-\omega t \cos (\omega t)}{\omega^{3}} \boldsymbol{g} \times \boldsymbol{\omega} \\
& +\frac{\omega t \sin (\omega t)-1+\cos (\omega t)}{\omega^{2}} \boldsymbol{\omega} \times\left(\boldsymbol{r}_{0} \times \boldsymbol{\omega}\right) \\
& -\frac{t(1-\cos (\omega t))}{\omega^{2}} \boldsymbol{\omega} \times\left(\boldsymbol{v}_{0} \times \boldsymbol{\omega}\right) \\
& +\frac{\omega t \sin (\omega t)-1-\omega^{2} t^{2} / 2+\cos (\omega t)}{\omega^{4}} \boldsymbol{\omega} \times(\boldsymbol{g} \times \boldsymbol{\omega})
\end{aligned}
$$

The solution presented in equation $(8)$ is the most general possible for the motion in the Earth's gravitational field with Earth in constant rotation. This result is quite different from what is obtained when omitting the contribution of the centrifugal terms in the equation of motion 2, as I will show.

\subsection{Coriolis only}

Considering only the noninertial contributions due to the Coriolis term, equation (1) becomes [4]

$$
\ddot{\boldsymbol{s}}=\boldsymbol{g}_{0}+2 \dot{\boldsymbol{s}} \times \boldsymbol{\omega}
$$

where the position vector was called $s$ to avoid confusion with the previous notation where centrifugal terms were used. Equation (9) is commonly solved in problems presented in Classical Mechanics lectures for Earth motion, 
but using a scalar approach, working with Cartesian coordinates separately [4. By following analogous steps to those shown in the previous subsection, one has the equation of motion

$$
\boldsymbol{s}^{(4)}+4 \omega^{2} \ddot{\boldsymbol{s}}=4 \omega^{2} \boldsymbol{g}_{0}-4 \boldsymbol{\omega} \times\left(\boldsymbol{g}_{0} \times \boldsymbol{\omega}\right)
$$

which is quite different from the higher-order equation of motion given in (5).

The solution of the differential equation 10 can also be obtained in an analogous way to that developed in the previous subsection, reaching the following result:

$$
\begin{aligned}
\boldsymbol{s}(t)= & \boldsymbol{r}_{0}+\boldsymbol{v}_{0} t+\frac{\boldsymbol{g}_{0} t^{2}}{2} \\
& +\frac{1-\cos (2 \omega t)}{2 \omega^{2}} \boldsymbol{v}_{0} \times \boldsymbol{\omega} \\
& +\frac{2 \omega t-\sin (2 \omega t)}{4 \omega^{3}} \boldsymbol{g}_{0} \times \boldsymbol{\omega} \\
& -\frac{2 \omega t-\sin (2 \omega t)}{2 \omega^{3}} \boldsymbol{\omega} \times\left(\boldsymbol{v}_{0} \times \boldsymbol{\omega}\right) \\
& +\frac{1-2 \omega^{2} t^{2}-\cos (2 \omega t)}{4 \omega^{4}} \boldsymbol{\omega} \times\left(\boldsymbol{g}_{0} \times \boldsymbol{\omega}\right)
\end{aligned}
$$

which is in agreement with the solution in terms of components found in the literature 44. The result obtained in 11) exhibits significant differences regarding the time dependence when compared to the solution given in (8). The comparison between the solutions given in (8) for the complete problem and (11) taking into account only the Coriolis force shows clearly that centrifugal terms cannot be discarded a priori. As an example of this difference in the temporal evolutions of the noninertial terms, Figure 1 shows the behavior of the temporal factors that multiply the vector product $\boldsymbol{v}_{0} \times \boldsymbol{\omega}$ of the accelerations of (8) and (11), given by $[2 \cos (\omega t)-\omega t \sin (\omega t)]$ and $2 \cos (2 \omega t)$, respectively.

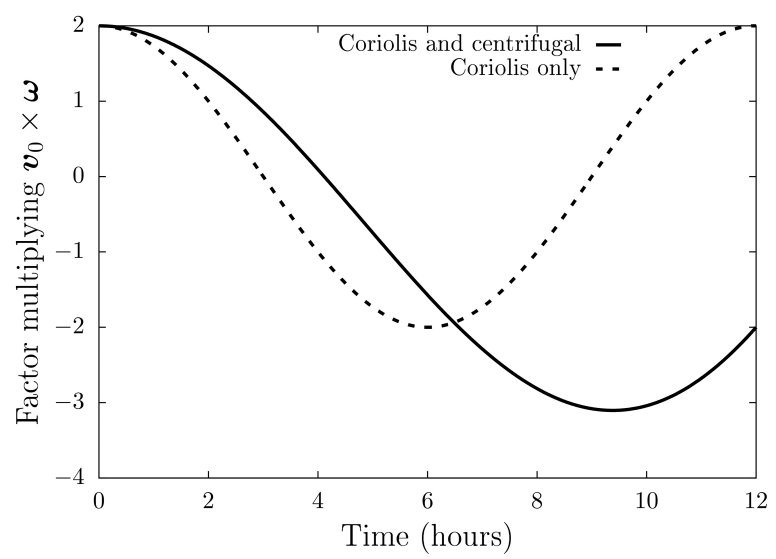

Figure 1: Comparison between the factors $[2 \cos (\omega t)-$ $\omega t \sin (\omega t)]$ and $2 \cos (2 \omega t)$ that multiply the vector product $\boldsymbol{v}_{0} \times \boldsymbol{\omega}$ for the accelerations of (8) and (11) obtained from the solution of the complete problem (solid line) and the problem with only Coriolis (dashed line).
It is known that the vector product $\boldsymbol{v}_{0} \times \boldsymbol{\omega}$ is responsible for several noninertial effects and, as Figure 1 clearly shows, to study these effects considering solely the Coriolis force, disregarding centrifugal contributions, can lead to misleading results in a matter of a few hours.

In the next section, I will show the noninertial effects according to the solution of the complete problem. Such effects will be classified according to the order of magnitude in $\omega$.

\section{Noninertial Effects}

To find the noninertial effects, it is necessary to evaluate the acceleration of the complete problem, given in equation (8). By differentiating it twice, it results

$$
\begin{aligned}
\ddot{\boldsymbol{r}}(t)= & \boldsymbol{g} \\
& +\omega(\sin (\omega t)+\omega t \cos (\omega t)) \boldsymbol{r}_{0} \times \boldsymbol{\omega} \\
& +(2 \cos (\omega t)-\omega t \sin (\omega t)) \boldsymbol{v}_{0} \times \boldsymbol{\omega} \\
& +\frac{\sin (\omega t)+\omega t \cos (\omega t)}{\omega} \boldsymbol{g} \times \boldsymbol{\omega} \\
& +(\cos (\omega t)-\omega t \sin (\omega t)) \boldsymbol{\omega} \times\left(\boldsymbol{r}_{0} \times \boldsymbol{\omega}\right) \\
& -\frac{2 \sin (\omega t)+\omega t \cos (\omega t)}{\omega} \boldsymbol{\omega} \times\left(\boldsymbol{v}_{0} \times \boldsymbol{\omega}\right) \\
& -\frac{\omega t \sin (\omega t)+1-\cos (\omega t)}{\omega^{2}} \boldsymbol{\omega} \times(\boldsymbol{g} \times \boldsymbol{\omega})
\end{aligned}
$$

Table 1 summarizes the order of magnitude of each term, taking into account its temporal factor.

To find the consequences of the noninertial effects, it is necessary to define the vectors $\boldsymbol{g}_{0}, \boldsymbol{R}$, and $\boldsymbol{\omega}$ from the reference frame located on Earth to find the final orientation of those vector products given in equation (12). The arrangement adopted for the axes of the noninertial coordinate system is given according to Figure 2

Table 1: Noninertial terms present in the acceleration of motion in the Earth's gravitational field with the Earth in constant rotation. Each noninertial term is given by the product of the temporal factor (first column) and the vector product (second column). The last column shows the order of magnitude in $\omega$ for each noninertial term present in the acceleration.

\begin{tabular}{lcc}
\hline Temporal factor & Vector product & $\mathcal{O}(\omega)$ \\
\hline $2 \cos (\omega t)$ & $\boldsymbol{v _ { 0 } \times \boldsymbol { \omega }}$ & 1 \\
$(\sin (\omega t)+\omega t \cos (\omega t)) / \omega$ & $\boldsymbol{g} \times\left(\boldsymbol{r}_{0} \times \boldsymbol{\omega}\right)$ & 2 \\
$\cos (\omega t)$ & $\boldsymbol{\omega} \times\left(\boldsymbol{v}_{0} \times \boldsymbol{\omega}\right)$ & 2 \\
$-(2 \sin (\omega t)+\omega t \cos (\omega t)) / \omega$ & $\boldsymbol{\omega} \times(\boldsymbol{g} \times \boldsymbol{\omega})$ & 2 \\
$-(\omega t \sin (\omega t)+1-\cos (\omega t)) / \omega^{2}$ & $\boldsymbol{v}_{0} \times \boldsymbol{\omega}$ & 3 \\
$-\omega t \sin (\omega t)$ & $\boldsymbol{r}_{0} \times \boldsymbol{\omega}$ & 3 \\
$\omega(\sin (\omega t)+\omega t \cos (\omega t))$ & $\boldsymbol{\omega} \times\left(\boldsymbol{r}_{0} \times \boldsymbol{\omega}\right)$ & 4 \\
$-\omega t \sin (\omega t)$ & & \\
\hline
\end{tabular}



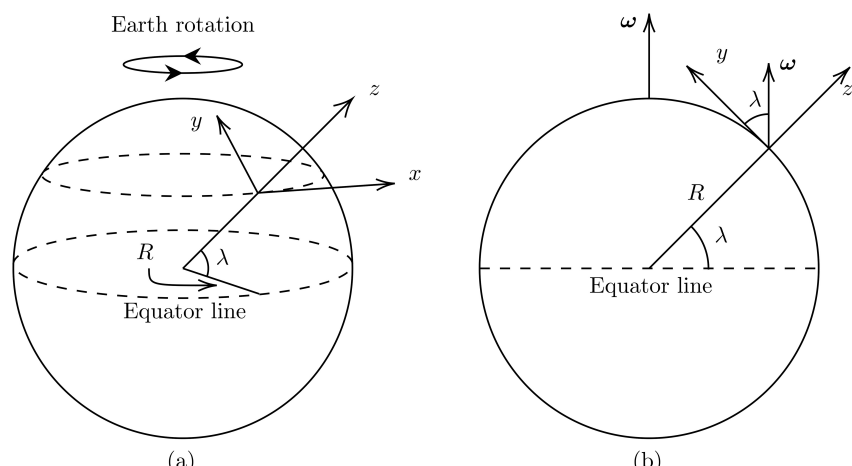

Figure 2: Scheme of the assumed coordinate system. In part (a) there is a perspective view while in part (b) there is a planned view. Latitude is determined by the angle $\lambda$.

Thus, according to the arrangement shown in Figure 2 the vectors $\boldsymbol{g}_{0}, \boldsymbol{R}$, and $\boldsymbol{\omega}$ are written as

$$
\boldsymbol{g}_{0}=-g \boldsymbol{e}_{z}, \quad \boldsymbol{R}=R \boldsymbol{e}_{z}, \quad \boldsymbol{\omega}=\omega\left(\cos \lambda \boldsymbol{e}_{y}+\sin \lambda \boldsymbol{e}_{z}\right)
$$

where $\boldsymbol{e}_{i}$ is the unit vector in the $i$ direction and $\lambda$ is the latitude, positive in the Northern Hemisphere. With the definitions given in (13), a Taylor expansion in $\omega$ to the second-order of 12 can be performed to specify noninertial effects for motion in the Earth's gravitational field with a constant rotating Earth. The results of such procedure are listed below:

- $\boldsymbol{v}_{0} \times \boldsymbol{\omega}$ - first-order.

- Deflection to the right (left) in the Northern (Southern) Hemisphere: $2 \sin (\lambda) \omega$ $\left(\dot{y}_{0} \boldsymbol{e}_{x}-\dot{x}_{0} \boldsymbol{e}_{y}\right)$

- Eötvös effect - the apparent weight of an object increases (decreases) as it travels to the west (east): $2 \dot{x}_{0} \cos (\lambda) \omega \boldsymbol{e}_{z}$.

- Ascending (falling) objects deviate to the west (east): $-2 \cos (\lambda) \dot{z}_{0} \omega \boldsymbol{e}_{x}$.

- $\boldsymbol{g} \times \boldsymbol{\omega}-$ first-order.

- Acceleration to the east. The closer to the Equator, the greater the force. This effect is null at the Poles: $2 g t \cos (\lambda) \omega \boldsymbol{e}_{x}$.

- $\boldsymbol{\omega} \times\left(\boldsymbol{v}_{0} \times \boldsymbol{\omega}\right)-$ second-order.

- Ascending (falling) objects deflect to the nearest Pole (Equator): $3 \dot{z}_{0} t \cos (\lambda)$ $\sin (\lambda) \omega^{2} \boldsymbol{e}_{y}$.

- Second-order Eötvös effect - the apparent weight of an object increases (decreases) as it travels towards the Equator (nearest Pole): $3 \dot{y}_{0} t \cos (\lambda) \sin (\lambda) \omega^{2} \boldsymbol{e}_{z}$.

- The apparent weight of an object increases (decreases) in ascending (descending) motion. This effect is maximum at the Equator, null at the Poles and independent on the hemisphere: $-3 \dot{z}_{0} t \cos ^{2}(\lambda) \omega^{2} \boldsymbol{e}_{z}$.
- $\boldsymbol{\omega} \times(\boldsymbol{g} \times \boldsymbol{\omega})-$ second-order.

- Acceleration oriented to the Equator: $-(3 / 2) g t^{2} \cos (\lambda) \sin (\lambda) \omega^{2} \boldsymbol{e}_{y}$.

- Reduction in apparent weight. This effect is maximum in the Equator, null at the Poles and independent on the hemisphere: $(3 / 2) g t^{2} \cos ^{2}(\lambda) \omega^{2} \boldsymbol{e}_{z}$.

where $\boldsymbol{v}_{0}=\left(\dot{x}_{0}, \dot{y}_{0}, \dot{z}_{0}\right)$.

None of the listed effects depend on the radius of the planet. With the exception of the corrective term $\boldsymbol{\omega} \times$ $(\boldsymbol{R} \times \boldsymbol{\omega})$ for the gravity field $\boldsymbol{g}$, only $\mathcal{O}\left(\omega^{3}\right)$ effects and superior depend on $R$.

The ratio between the contributions of third-order to the first-order and of fourth-order to the secondorder that appear in the same acceleration components has a modulus $R \omega^{2} / g$, which in the case of Earth corresponds to about $0.35 \%$. Thus, $\mathcal{O}\left(\omega^{3}\right)$ and $\mathcal{O}\left(\omega^{4}\right)$ effects were omitted from the description due to their low contribution compared to the first and secondorder effects, respectively. This result coincides with the semiquantitative comparison between the centrifugal term $\boldsymbol{\omega} \times(\boldsymbol{R} \times \boldsymbol{\omega})$ (second-order) and the module of the terrestrial gravitational field $\boldsymbol{g}_{0}$ (zeroth-order) [1, 3]. Furthermore, the $\mathcal{O}\left(\omega^{3}\right)$ and $\mathcal{O}\left(\omega^{4}\right)$ effects have the same orientation, given by the vector term, as the $\mathcal{O}(\omega)$ and $\mathcal{O}\left(\omega^{2}\right)$ effects, respectively, as seen in Table 1, meaning that they compete with each other and hence the loworder effects (first and second) tend to suppress highorder effects (third and fourth).

It is possible to notice that, in general, the first-order effects manifest in the east-west direction, while the second-order ones manifest in the north-south direction. Therefore, these two classes of effects do not compete with each other.

The theoretical explanation of higher-order effects in Physics is very important once new phenomena can arise from them. Here several second-order effects for the motion in rotating Earth were shown and such findings can certainly contribute to the deepening of Physics classes as well as a theoretical background for basic research concerning the observation of such phenomena.

\section{Conclusions}

The motion in the gravitational field evaluated by a noninertial frame fixed to the Earth in constant rotation was studied analytically without carrying out a priori approximations, that is, taking into account both the Coriolis and centrifugal terms for the composition of the motion. The decoupling of the equation of motion was performed, producing a linear sixth-order differential equation. The analytical solution of the decoupled equation was obtained for generic initial conditions, showing all noninertial terms. The same procedure was performed for the motion considering only the Coriolis term and ignoring the centrifugal one from the start. 
As a result of the comparison of the two procedures, it was found that the decoupled differential equations are very different from each other, leading to very different solutions, although there is a certain coincidence in the description of the consequences of the noninertial effects. However, this coincidence only occurs because the two solutions are very close to each other when a short time elapses, but differ significantly for longer periods.

The consequences of noninertial terms for the motion were also presented, ranked according to the order of magnitude in $\omega$, the (angular) frequency of Earth rotation. In addition to the commonly known consequences such as lateral deviation and the Eötvös effect, which are first-order, several second-order effects have been described, being oriented in a north-south direction. Therefore there is no direct competition with the firstorder effects, oriented in an east-west direction.

\section{References}

[1] S.T. Thornton and J.B. Marion, Classical Dynamics of Particles and Systems (Cengage Learning, Belmont, 2004).

[2] D. Morin, Introduction to Classical Mechanics: With Problems and Solutions (Cambridge University Press, Cambridge, 2008).

[3] H. Goldstein, C.P. Poole and J.L. Safko, Classical Mechanics (Addison Wesley, San Francisco, 2002).

[4] W. Greiner, Classical Mechanics: Systems of Particles and Hamiltonian Dynamics (Springer, New York, 2003). 